\title{
How Chinese Medicine functions on COVID-19, based on three Chinese Medicine prescriptions with 220-1800 years history
}

\author{
lixing liu ${ }^{1}$ \\ ${ }^{1}$ Chinese Academy of Medical Sciences and Peking Union Medical College
}

June 17,2020

\begin{abstract}
COVID-19 caused a worldwide pandemic and he therapeutic effect of traditional Chinese medicine is exciting and promising, but the unclear biological mechanism and culture differences have led to its limited acceptance and application. This study selected three basic traditional Chinese medicine prescriptions for fighting epidemics with 200-1800 years application history and applied systematic network pharmacology to clarify their biological mechanism. We found that: (1) Five compounds that played a key role in treating virus pneumonia were screened out and showed well molecular docking efficiency with COVID-19. And the core hub gene of all three prescriptions was RB1. (2) In terms of function, the predicted targets of three Chinese medicine prescriptions were abundantly expressed in regulating viral replication cycle, regulating immune and inflammatory response, coagulation function, and cell proliferation functions. (3) Three prescriptions with completely different properties and different herbs showed very similar biological functions, and the same compound may affect the expression of pathway genes in the same or opposite directions, which reflected the two-way regulatory characteristics. This study suggested that Chinese medicine had great potential in the regulation of viral pneumonia cell cycle and immune microenvironment, and indicated the common biological basis of Chinese medicine in treatment of COVID-19.
\end{abstract}

\section{Introduction}

The coronavirus infected millions of people and caused a global pandemic. It was reported that the basic reproduction number $\mathrm{R}(0)$ of this infectious disease was 2.2, or even up to 6.6 [1-2]. About $20.9 \%$ of the confirmed diagnosis patients had no symptoms [3] that also made the new coronavirus more hidden and spread rapidly. For a while, the virus is rampant due to the strong infectivity and lack of effective antiviral drugs. Therefore it is urgent to develop effective drugs and vaccines. However, it will take quite a long time and huge efforts to develop vaccines and drugs that may miss the epidemic situation and the virus even may mutate. Chinese medicine that is symptom-oriented medicine was highlighted in this virus campaign in China by delaying the progress of the disease, reducing the sufferings of patients, and preventing the spread of the disease. There were clinical observations reported from Wuhan that integrated Chinese medicine and Western medicine could improve clinical symptoms two days faster than western medicine alone and cure fever in 2 days. And the improvement rate of CT image was $88.2 \%$, the clinical cure rate was $94.1 \%$ and $5.9 \%$ of the general type progressed to the severe type, which were significantly better than those of the western medicine group $(53.8 \%, 68.8 \%, 61.1 \%, 33.3 \%$, respectively)[4]. Notably, the mortality rate of this clinical observation is only $2 \%$, lower than that of the whole mortality rate in Wuhan area. This showed that the integration of Chinese and Western treatment could reduce the progress of novel coronavirus pneumonia $(\mathrm{NCP})$, promote the recovery of severe and critical patients, and reduce mortality. In the future, more data reports on clinical research of TCM treatment are expected. From the perspective of modern medicine, it's hard to make the complex mechanism clear since there are many components in Chinese herb remedies.

Recently, many scholars have adopted modern methods to explore its action mechanism. However, there are many kinds of prescriptions actually used in clinical, which are individualized treatment according 
to the symptoms of patients rather than some fixed herbs. TCM recognizes diseases from the clinical manifestations of cold and hot symptoms of patients and chooses the prescription of anti-drug to treat. Simply, for example, the cold syndrome is treated by the herbs with warm or hot quality, the hot syndrome by the herbs with cold feature, and the damp syndrome treated by the herbs with dry character. We chose three kinds of clinically proven representative prescriptions according to the three different syndromes of the NCP. Then the Ma Huang decoction (cold syndrome), Yin Qiao powder (hot syndrome) and Da Yuan Yin decoction (damp syndrome) that were adopted in the treatment of NCP were selected. These three prescriptions are representative of the clinical experience of thousands of years in clinical application. To systematically elucidate the same disease treated with different herb prescriptions, we carried out the network pharmacological analysis to find out the universal biological mechanism behind the curative effect.

\section{Methods:}

Representative prescriptions selection and database construction

The representative prescriptions were selected according to the following principles:(1. Basic prescriptions commonly used in treating viral pneumonia with hundreds or thousands of year's clinical practice; 2. Classical prescriptions recorded in ancient Chinese medicine books; 3 . The treatment effect is accurate, supported by relevant literature and books; 4. Select the representative prescriptions of different TCM Etiology and pathogenicity of diseases). And finally the representative prescriptions were chosen as bellow: the cold syndrome: Mahuang decoction (ephedra herb (Guizhi), almond, liquorice), heat syndrome: Yinqiao Powder (honeysuckle, forsythia, bamboo leaf, Schizonepeta, burdock, light Douchi, mint, liquorice, Platycodon grandiflorum, reed root), damp syndrome: Dayuan decoction (areca, Magnolia officinalis, Cao Guo, Anemarrhena, peony, Scutellaria, liquorice). The chemical components of each traditional Chinese medicine in each prescription were retrieved from several TCM datasets, including TCMSP[5], PubMed (https://pubmed.ncbi.nlm.nih.gov/), CNKI (https://www.cnki.net/), NCBI PubChem (https://pubchem.ncbi.nlm.nih.gov/) databases, etc. The repeated components were deleted and then the components database was eatablished. Viral pneumonia (VP) related targets were collected through Genecards [6], OMIM [7] and DisGeNET [8] databases. In the Genecards database, those with scores greater than 5 points were selected as potential disease targets.Active ingredients screen and drug targets predictionThe active chemical components were screed according to the oral bioavailability (oral bioavailability, OB $>30 \%$ ) and drug like (drug likeness, DL > 0.18) from the TCMSP database [9-10]. Then the information of chemical components of each prescription was finally obtained (Table 1). The parameters are predicted by OBioavail model, Tanimoto coefficient and PreDHL model respectively [11-12]. The corresponding 2D structures of the active ingredients were retrieved by PubChem database, and the 2D structure diagrams were drawn by using Chemdraw. Drug targets were collected by drugbank and pharmmapper database [1314]. Drug target prediction model SysDT and drug pharmacophore models were used to predict compound related targets [15-16]. The targets were standardized and limited with human species through UniProt database [17].Network establishment and enrichment analyses

Venn diagram was used to draw Venn map that visualized the intersection of the active herb components and disease targets. Herb compounds and disease targets action network were made through the software Cytoscape and the topological properties, maximal clique centrality (MCC) of the network are analyzed by using Cytohubba [18-19]. From a systematic point of view, we studied the molecular mechanism of diseases, find the target of active herb conpounds, and to explore the core genes of treatment of diseases through the protein-protein interaction (PPI) finished by string 11.0 database [20]. In order to verify that the potential target of the selected prescription is closely related to viral pneumonia, the enrichment analyses of GO and KEGG were carried out by R package cluster Profiler and the cluster analysis by Cluego [21-22]. The biological process and molecular function enrichment analysis of the intersection gene were carried out to study the pathway of active herb components targets. And the BINGO plug-in was applied to draw the Directed acyclic graph (DAG) of intersection genes and analyze the low-level functional enrichment results in each graph [23]. Finally, a visualized and integrated disease and function network of herb compounds targets pathway is constructed. 


\section{Results}

Herbs-disease intersection genes and key effective compounds

A total of 976 compounds were collected through TCMSP , Pubmed and CNKI database searching for MH Decoction, a total of 1177 compounds were collected in YQ Powder, and a total of 839 compounds were collected in DY Decoction. And the all these compounds were screened to obtain the active ingredients by the standard of OB [?] $30 \%$ and DL [?] 0.18. Finally, MH Decoction collected a total of 116 effective compounds, and obtained 257 function-related targets. A total of 133 active ingredients were collected in YQ Powder, and there were 278 targets related to it were obtained (Supplement material1-2). A total of 145 effective compounds were collected in DY Decoction, and 264 targets related to Dayuanyin were harvested (Figure 1A). A total of 983 targets were collected as potential disease targets through OMIM, DisGeNET and Genecards databases. Venn diagram was obtained after the effective compounds and disease targets were intersected. 117 intersections targets between $\mathrm{MH}$ decoction and disease were found. There were 125 intersections of YQ powder and disease. And there were 118 targets for the intersection of DY decoction and disease (Figure1B). By comparing the number of interconnections between drug-disease intersection genes and randomly selected genes, it is shown that the drug-disease intersection genes we obtained are biologically interconnected (Figure1C).

Herb compounds and disease targets action network were shown in Figure 1D. As it shown, MH decoction, YQ powder, and DY decoction obtained 234, 262, and 259 nodes, with 1227, 1388, and 1424 edges respectively. The network density was $0.045,0.041$, and 0.043 , and the network diameter was $5,6,5$ respectively. The network heterogeneity was $1.558,1.586$ and 1.609 ; with network centralization was $0.392,0.395,0.434$ respectively. And average number of neighbors was 10.487, 10.595 and 10.996 respectively (Figure1D). According to the disease target network diagram of each prescription compounds, the key five compounds for viral pneumonia of each prescription were found. They were key compound as quercetin (MOL000098), luteolin (MOL000006), kaempferol (MOL000422), naringenin (MOL004328), and licochalconea (MOL000497) for Mahuang decoction to treat viral pneumonia. And quercetin (MOL000098), luteolin (MOL000006), kaempferol (MOL000422), wogonin (MOL000173), and naringenin (MOL004328) were the key compound of YQ powder. And for DY decoction, the five key compounds for treatment of viral pneumonia were quercetin (MOL000098), kaempferol (MOL000422), wogonin (MOL000173), naringenin (MOL004328), and licochalconea (MOL000497)(Figure 1E).

RB1 was the core genes of all three herbs prescriptions that mainly functioned as negative regulation of G1/S transition of mitosis cell cycle

The intersection genes were imported into the string database and a PPI network graph were drawn. Among them, the number of nodes obtained by MH decoction and DY decoction were both 101, and YQ powder was 111. There are 448, 514 and 454 sides for MH, YQ and DY PPI network respectively (Figure 2AC). Cytohubba plug-in was used to calculate the central genes in the network and to map the central gene interaction network (Figure 2D-F). Among them, the top ten Hub genes of MH decoction were RB1, E2F1, CDKN1A, CDK2, CDK1, CDK4, CCND1, PCNA, MAPK3 and JUN. The top 10 Hub genes for YQ powder were RB1, E2F1, CDKN1A, CDK2, CDK1, CDK4, CCND1, PCNA, MAPK3, MAPK1. And for DY decoction's top ten were RB1, CDKN1A, MAPK3, E2F1, CDK4, CDK2, CDK1, MAPK1, RELA and JUN (Figure 2 G-I). Among them, the core genes of MH and YQ were both RB1. The core genes of DY were RB1 and CDKN1A. By Go rich clustering analysis the hub genes, the results showed that the hub gene functions of $\mathrm{MH}$ decoction were mainly concentrated in the negative regulation of $\mathrm{G} 1$ / S transition of mitosis cell cycle $(65.63 \%)$, the regulation of cell cycle arrest $(29.69 \%)$, positive regulation of fibroblast proliferation(3.12\%)and regulation of transcription involved in G1/S transition of mitotic cell cycle $(1.56 \%)$ (Figure3A). The hub of gene function of YQ powder are mainly concentrated in negative regulation of G1/S transition of mitotic cell cycle(56.72\%), regulation of cardiac muscle cell proliferation(22.39\%),regulation of cell cycle arrest(17.91\%), positive regulation of fibroblast proliferation(1.49\%)and regulation of transcription involved in G1/S transition of mitotic cell cycle(1.49\%)(Figure3B). The hub of gene function of DY decoction mainly focused on the negative regulation of G1/S transition of mitotic cell cycle(82.76\%), cellular response 
to reactive oxygen species(10.34\%)and regulation of fibroblast proliferation(6.9\%)(Figure3C). All three had the effect of negatively regulating the transition of mitotic G1 phase to S phase and positively proliferating fibroblasts. Both $\mathrm{MH}$ decoction and YQ powder have the effect of regulating cell cycle arrest, and YQ also plays a role in cardiomyocyte proliferation, and DY decoction showed a regulation function in respond to reactive oxygen species.

The three prescriptions showed participation in the whole pathological processes after the virus invaded the human body

The Go biological process enrichment was carried out for all the intersection genes, among which $\mathrm{MH}$ decoction obtained a total of 2448 entries, YQ powder obtained a total of 2563 entries, and DY decoction obtained a total of 2558 entries. According to the consistency cluster analysis of Go function of all the intersecting genes with kappa value of $0.3, \mathrm{MH}$ decoction has 13 categories, such as regulation of never generation (42.38\%), regulation of immature $\mathrm{T}$ cell promotion (10.6\%), cell response to insulin like growth factor stimulus $(8.61 \%)$, etc.(Figure $4 \mathrm{~A}$ ). There are 16 categories of YQ powder, including regulation of establishment of endothelial barrier $(38.37 \%)$, regulation of immature T cell promotion $(9.88 \%)$, positive regulation of MHC (major histocompatibility complex, MHC) class II biosynthetic process (7.56\%), etc. (Figure 4B). There are 13 categories of DY decoction, containing regulation of fever generation (47.71\%), regulation of immature $\mathrm{T}$ cell promotion $(17.71 \%)$, connective tissue replacement involved in inflammatory response wound healing (8\%), etc. (Figure $4 \mathrm{C})$.

In the virus replication cycle, it usually experiences the process of adsorption, penetration, biosynthesis, assembly and release. All three prescriptions have functions on cellular response to virus (GO: 0098586), virtual entry into host cell (GO: 0046718), regulation of viral genome replication (GO: 0045069), regulation of protein complex assembly (GO: 0043254), and also regulation of viral life cycle (GO: 1903900), which affects the entire process of the virus replication cycle and comprehensively fight the virus infection of the cell. In regulating immune response and inflammation, the three prescriptions showed the functions on response to oxidative stress (GO: 0006979), reactive oxygen species metabolic process (GO: 0072593), lymphocyte proliferation (GO: 0046651), phagocytosis (GO: 0006909), etc. Through these processes, the three decoctions regulated immune system to promote the killing of viruses, and reduced the damage to the body during these processes by functioning on the regulation the infiltration of inflammatory factors, oxidative stress, active oxygen metabolism and blood coagulation (Table 1).

In order to explore the specific function of each prescription in the biological process, we have drawn the Directed Acyclic Graph of the three prescriptions and analyzed the nodes at the lower level. The three prescriptions were all have the function of regulating the proliferation of $\mathrm{T}$ cells and $\mathrm{B}$ cells in both positive and negitive directions (Figure 5). They could promote $\mathrm{B}$ and $\mathrm{T}$ cell proliferation and differentiation, positive regulation of immunoglobulin secretion, antigen presentation and co-stimulation. And at the same time, they could also induce B cell apoptosis and avoided T cell excessive activation, which maintaining B and T cell homeostasis. In innate immunity, all three showed the effect of promoting innate immune response in mucosa, promoting leukocyte tethering or rolling, macrophage differentiation, and regulating regulation of leukocyte mediated cytotoxicity, in order to achieve defense response to virus and avoid evasion of host defenses by virus.

In the regulation of cytokines and inflammation, the three prescriptions positively regulated the synthesis of IL-2, IL-6, IL-12 and leukotriene, and promoted leukocyte migration involved in inflammatory response and positively regulated cytokine secretion, connective tissue replacement, wound healing and fever. Meanwhile, they also showed negative regulation of inflammatory response to avoid the storm of inflammatory factors caused by excessive inflammatory response. At the same time, the three also showed functions of regulating the cell cycle, promoting angiogenesis and coagulation, and affecting the development of the alveoli. In regulating the cell cycle, they have the role of promoting positive cell cycle arrest (positive regulation of cell cycle arrest - negative regulation of mitotic cell cycle), activate DNA damage checkpoints (DNA damage response, signal transduction by p53 class mediator - G1/S DNA damage checkpoint - mitotic cell cycle G2/M transition DNA damage checkpoint) for each replication cycle. In terms of coagulation, they 
demonstrated negative regulation of plasminogen activation, positive regulation of platelet-derived growth factor receptor signaling pathway, and regulation on platelet activation to maintain the steady state of coagulation function. The three also played roles in regulating cellular response to hypoxia in hypoxia, and promote lung alveolus development.

In addition to the similar functions mentioned above, the three also showed their own characteristics. MH decoction showed positive regulation of protein complex assembly. YQ powder played a role in regulating $\mathrm{T}$ cell differentiation in the thymus and mesenchymal cell proliferation in lung development. DY decoction demonstrated positive regulation of respiratory burst involved in inflammatory response, positive regulation of toll-like receptor 4 signaling pathway, defense response to gram-positive bacterium, regulating activation of myeloid leukocyte, Rac GTPase activity, and negative regulation of macroautophagy, which showed prominent function on defense virus and immune activation.

Three prescriptions concentrated in KEGG pathway showed close relationship with infection and tumor disease

After enrichment of KEGG pathway, 162 pathways were found in each prescription, and the top 20 entries were plotted as barplot (Figure6), which referred mainly with infection, tumor, cardiovascular diseases and endocrine diseases. In the treatment of viral pneumonia, the three decoctions mainly rely on affecting the pathways in Table 2, such as viral protein interaction with cytokine and cytokine receptor (hsa04061), apoptosis (hsa04210), antigen processing and presentation (hsa04612), B cell receptor signaling pathway (hsa04662), T cell receptor signaling pathway (hsa04660), natural killer cell mediated cytotoxicity (hsa04650) and so on (Table 2). The top five compounds that most closely affected these pathways were: quercetin (MOL000098), luteolin (MOL000006), kaempferol (MOL000422), wogonin (MOL000173), and naringenin (MOL004328). Among them, quercetin, kaempferol and naringenin were the common compounds of the three prescriptions. These five key compounds are derived from the ephedra in Mahuang decoction; the honeysuckle and forsythia extract in Yinqiao powder, the tsaoko and scutellaria baicalensis in Dayuan decoction. Interestingly, these herbs were just the key herbs in each prescription according to the theory of traditional Chinese medicine, which also proves the rationality of the theory of traditional Chinese medicine. The therapeutic mechanisms of these five compounds were that they could affect apoptosis, adaptive immunity and innate immunity (Figure 7). RB1as the common hub gene of the three prescriptions, and through the query of the KEGG pathway related with RB1, we found that it mainly enriched in viral infections and tumors, of which viral infections were mainly concentrated in Hepatitis B (hsa05161), Hepatitis C (hsa05160), Epstein-Barr virus infection (hsa05169) and other 8 pathways. The tumors are mainly enriched in 10 pathways including lung tumors, digestive system tumors, and urogenital system tumors. These findings indicated that the three prescriptions were most closely related to infection and tumor diseases.

The five compounds have the potential as a new target drug for covid-19

The 3C-like protease (3CL) of COVID-19 can cut the precursor protein of the viral genome translation, and these cleaved non-structural proteins form the replicase-transcriptase complex. Therefore, 3CL is a very important drug target in the process of virus reproduction.

Among the five compounds we have obtained previously, all of them can bind to 3CL stably through Conventional Hydrogen Bond and hydrophobic interaction (Pi-Pi T-shaped and Pi-Alkyl). And Kaempferol (Figure 8A), Luteolin (Figure $8 \mathrm{~B}$ ) and Naringenin (Figure $8 \mathrm{C}$ ) can also form unconventional Hydrogen Bond (Pi-Donor Hydrogen Bond or Carbon Hydrogen Bond) to enhance the stability of the bond. While, there are pi-sulfur interactions in Kaempferol, Naringenin and Quercetin (Figure 8D), which is a kind of the metal-acceptor interaction and make the binding more stable. Kaempferol and Wogonin (Figure 8E) can also form Pi-sigma forces, which increased the stability of the binding of macromolecular proteins and small molecular compounds in aqueous solvents. However, there is still a Donor-Donor Clashes for Luteolin docking, which influenced the stability of the binding of compounds with pathogens targets. In the study of ligands of 3CL (Figure $8 \mathrm{~F}$ ), the binding efficiency of the five compounds found in this paper is close to that of ligands of 3CL itself. At the same time, we calculated the inhibition constant (Ki) of the compound to 
3CL at 298.15k. With the decrease of docking energy, the inhibition constant also decreased (Table 3). In general, these compounds can bind closely to the 3c-like proteases of covid-19, which have the potential as a new target drug for covid-19.

\section{Discussion}

A total of 2,992 compounds of three prescriptions were collected and 394 effective compounds were selected after activity screening. There are 983 potential targets for disease treatment. After intersecting effective compounds with disease targets, 117 targets for $\mathrm{MH}, 125$ targets for YQ and 118 targets for DY were obtained respectively. The disease and herbs compounds target network showed that RB1 was the core hub gene shared by the three prescriptions. The cluster analysis of go enrichment of hub gene showed that the three prescriptions had a negative regulation of the transition of mitotic G1 phase to $\mathrm{S}$ phase, and positive fibroblast proliferation. Additionally, $\mathrm{MH}$ and $\mathrm{YQ}$ had the function of regulating cell cycle arrest; YQ also displayed ability of regulating cardio-myocyte proliferation; DY showed the reaction to the reactive oxygen species. Enrichment analysis of Go biological processes was carried out on all intersection genes. All three prescriptions could affect the whole process of virus replication cycle by inhibiting virus adsorption on cells, virus penetration, virus synthesis, and virus assembly and release, and play roles in regulating immune response and inflammatory response. The results showed that the three prescriptions could regulate the infiltration of inflammatory factors, oxidative stress and metabolism of reactive oxygen species, so as to protect the body and avoid lung injury caused by reactive oxygen species in the process of killing the virus. And they could improve the patient's fever symptom and adjust blood coagulation function. At the low level of functional concentration analysis, the three prescriptions showed the function of bidirectional regulation. On one side, they could promote the proliferation, differentiation, antigen presentation and co-stimulation of immune cells; on the other side, they could avoid their over-activation and prevent the over-reaction of immune response. There are 162 pathways enriched by KEGG analysis, which were closely related to infection and tumor diseases. The top five compounds that affect these pathways are quercetin (mol 000098), luteolin (mol 00006), kaempferol (mol 000422), wogonin (mol 000173), naringenin(MOL004328). Among them, quercetin, kaempferol and naringenin are compounds common to the three prescriptions. These compounds can bind closely to the 3c-like proteases of covid-19, showing the potential to become covid-19 targeted drugs[Figure 9].

When the virus invades through the cell surface receptors, it was recognized by the antigen presenting cells and presented to CD4 $+\mathrm{T}$ cells. B cells can directly recognize CD4 $+\mathrm{T}$ cells, and then secrete IgM and IgG to resist virus invasion [24]. COVID-19 related lymphocytopenia was a manifestation of disease exacerbation, and also showed the high vulnerability of lymphocytes to infection and destruction caused by COVID-19 [25,26]. The obvious reduction of regulatory $\mathrm{T}$ cells led to the occurrence of excessive inflammation and destroying the immune homeostasis [27]. At present, the mechanism leading to lymphocyte reduction is not clear. Some studies indicated that this might be related to virus-induced lymphocyte apoptosis and/or bone marrow damage caused by cytokine storms [25]. COVID-19 activated macrophages to release pro inflammatory cytokines and the toll-like receptor pathway plays an important role in virus-induced inflammation [28-29]. In some severe COVID-19 infected patients, systemic inflammation was observed [30], and IL-6, IL-2, IL-7, and TNF- $\alpha$ increased significantly with high mortality prognosis [31]. In addition, 7 to 14 days after the virus infection, patients began to appear hypercoagulable state [32]. Studies have shown that pro-inflammatory factors such as TNF- $\alpha$, interleukin, etc., can strongly induce the expression of tissue factor (TF) on the surface of monocytes, resulting in a hypercoagulable state of the body [33]. Then lung injury followed by the above pathological process, and acute lung injury was the main cause of death caused by covid-19. It was reported that related to inflammatory outbreak and release of proinflammatory mediators. Fibrin accumulated in the alveoli, which reduced oxygen exchange and ultimately led to respiratory distress syndrome [34]. Autopsy showed that bilateral diffuse alveolar injury was accompanied by cellular fibromyxoid exudation and interstitial mononuclear inflammatory infiltration, suggesting that exudative inflammation was closely related to the lung injury caused by covid-19 [35]. It's reported that heart injury occurred in $20-30 \%$ of patients, and caused $40 \%$ of patients die [36]. The mechanism of heart injury is not yet clear. Some studies have suggested that vasodilation, endothelial permeability and inflammatory process after 
leukocyte recruitment caused by respiratory failure and virus infection lead to cardiovascular stress and eventually myocardial injury [37]. In this study, it was found that the three prescriptions can maintain immune homeostasis, body temperature homeostasis and promote injury repair. They played a regulatory role in the entire disease process and presented a two-way regulatory feature. And the three prescriptions all have the effect of regulating hypoxia and the development of lung acinar. YQ also played a role in the proliferation of cardiomyocytes, preventing the virus from attacking heart. Moreover, the three can also regulate the stability of coagulation function and slow down the damage of important organs. It's reported that in patients who died at the age of 60 and over, new coronary pneumonia often occurs simultaneously with chronic diseases such as tumor diseases [34]. Interestingly, the three also played a role in tumor treatment pathways.

The study is also trying to demonstrate the core theory of traditional Chinese medicine that is syndrome differentiation in the treatment of diseases in a modern way. Syndrome differentiation is to diagnose the cold, heat, or damp characteristics of the same disease according to the symptoms that different patients with different constitutions showed. Then the herbs with relatively cold or heat resistance properties are selected, reflecting the same disease with different treatment. Interestingly, we found that although the compositions of the three herbal prescriptions were completely different, the functions, the targets and pathways that affect the disease were the same. We also found that the hub gene RB1, as a common gene to the three prescriptions, was mainly enriched in viral infections and tumors, which was also in line with our actual clinical practice. Because the theory of treating diseases by traditional Chinese Medicine is based on the symptoms of a single patient, so at a certain stage for different diseases, patients showed similar symptoms and then the same kind of herb prescriptions will be chosen. This is another core feature of traditional Chinese medicine theory that different diseases are treated with the same. Taking this covid-19 disease as an example, we introduced the basic theory of TCM to treat diseases, and used network pharmacology to reveal the internal biological mechanism of the theory.

\section{Conclusion}

In the present study, we integrated a series of methods to conduct a network pharmacology analysis of the theory and biological mechanism of traditional Chinese medicine treating COVID-19. We conclude that Chinese herbal medicine may treat covid-19 by regulating the cell cycle and immune-related pathways. The five key compounds quercetin, luteolin, kaempferol, wogonin, and naringenin which are origin from ephedra, honeysuckle, forsythia and grass fruit have the functions of regulating various biological processes such as inflammation, immunity, apoptosis, and blood coagulation, and exhibited the characteristics of bidirectional regulation. And importantly, the five compounds showed good docking effects with covid19. As a common target gene of three different prescriptions, Rb1 demonstrated the biological essence of Chinese Medicine treatment of the same disease. In addition, compounds from one same herb showed similar or different regulatory pathways, suggesting the role of TCM prescriptions in regulating the overall functions of pathogens and the host environment. To sum up, this study provided the biological mechanism of TCM for the treatment of covid-19, and found that five compounds showed the potential to be developed into targeted drugs for COVID-19.

Author Contributions: Conceptualization and writing: L.L; Network pharmacology analysis: J.CH; Supervision L.F. Lixing Liu and Jinyuan Chang contributed equally in this study.

Ethics approval and consent to participate: Not applicable.

Funding: This study was funded by China Postdoctoral Science Foundation Grant (Grant number: 2019M650575) ; Beijing Science and Technology Commission "Research and Development of Ten Diseases and Ten Drugs" Project (Grant number: Z171100001717017).

Acknowledgments: Thanks to the medical workers all over the world for their sacrifices and contributions in the fight against the COVID-19 pandemic.

Conflicts of Interest: The authors declare no conflict of interest. 


\section{References}

[1] Special Expert Group for Control of the Epidemic of Novel Coronavirus Pneumonia of the Chinese Preventive Medicine Association. An Update on the Epidemiological Characteristics of Novel Coronavirus pneumonia(COVID-19)Zhonghua Liu Xing Bing Xue Za Zhi, 2020 Feb 1441 (2), 139-144. DOI: $10.3760 /$ cma.j.issn.0254-6450.2020.02.002

[2]Steven Sanche, Yen Ting Lin, Chonggang Xu, et al.The Novel Coronavirus, 2019-nCoV, is Highly Contagious and More Infectious Than Initially Estimated. MedRxiv[Preprint]. Feb 11, 2020 [cited 2020 Mar.12]. doi: Available from: https://doi.org/10.1101/2020.02.07.20021154

[3] Wei-jie Guan, Zheng-yi Ni, Yu Hu, et al.Clinical characteristics of 2019 novel coronavirus infection in China. New England Journal of Medicine. 2020,February 28, DOI: 10.1056/NEJMoa2002032.

[4] Wenguang Xia, Changqing An, Chanjuan Zheng, et al. Clinical study on 34 novel coronavirus pneumoniae treated with integrated traditional Chinese and Western Medicine. Journal of Traditional Chinese Medicine. 2020, Feb 18. Online ahead of print

http://kns.cnki.net/kcms/detail/11.2166.R.20200217.1502.004.html

[5]RU J, LI P, WANG J, et al. TCMSP: a database of systems pharmacology for drug discovery from herbal medicines[J]. J Cheminform, 2014,6: 13.

[6]STELZER G, ROSEN N, PLASCHKES I, et al. The GeneCards Suite: From Gene Data Mining to Disease Genome Sequence Analyses[M]. John Wiley \& Sons, Ltd, 2016: 1-30.

[7]HAMOSH A, SCOTT A F, AMBERGER J, et al. Online Mendelian Inheritance in Man (OMIM)[J]. Hum Mutat, 2000,15(1): 57-61.

[8]PINERO J, RAMIREZ-ANGUITA J M, SAUCH-PITARCH J, et al. The DisGeNET knowledge platform for disease genomics: 2019 update[J]. Nucleic Acids Res, 2020,48(D1): D845-D855.

[9]XU X, ZHANG W, HUANG C, et al. A novel chemometric method for the prediction of human oral bioavailability[J]. Int J Mol Sci, 2012,13(6): 6964-6982.

[10]TAO W, XU X, WANG X, et al. Network pharmacology-based prediction of the active ingredients and potential targets of Chinese herbal Radix Curcumae formula for application to cardiovascular disease[J]. J Ethnopharmacol, 2013,145(1): 1-10.

[11]Xu X,Zhang W,Huang C,et al.A Novel Chemometric Method for the Prediction of Human Oral Bioavailability.International Journal of Molecular Sciences,2012,13(6):6964-6982

[12]Yang H,Zhang W,Huang C,et al.A novel systems pharmacology model for herbal medicine injection: a case using Reduning injection.BMC Complementaryand Alternative Medicine,2014,14(1):430

[13] WISHART D S, FEUNANG Y D, GUO A C, et al. DrugBank 5.0: a major update to the DrugBank database for 2018[J]. Nucleic Acids Res, 2018,46(D1): D1074-D1082.

[14]WANG X, SHEN Y, WANG S, et al. PharmMapper 2017 update: a web server for potential drug target identification with a comprehensive target pharmacophore database[J]. Nucleic Acids Res, 2017,45(W1): W356-W360.

[15] $\mathrm{Yu} \mathrm{H,Chen} \mathrm{J,Xu} \mathrm{X,et} \mathrm{al.A} \mathrm{systematic} \mathrm{prediction} \mathrm{of} \mathrm{multiple} \mathrm{drugtarget} \mathrm{interactions} \mathrm{from} \mathrm{chemical,}$ genomic, and pharmacological data.Plos One,2011,7(5):e37608

[16]LIU X, OUYANG S, YU B, et al. PharmMapper server: a web server for potential drug target identification using pharmacophore mapping approach[J]. Nucleic Acids Research, 2010,38(Web Server): W609-W614.

[17]UniProt: a worldwide hub of protein knowledge[J]. Nucleic Acids Res, 2019,47(D1): D506-D515. 
[18]SHANNON P, MARKIEL A, OZIER O, et al. Cytoscape: a software environment for integrated models of biomolecular interaction networks[J]. Genome Res, 2003,13(11): 2498-2504.

[19]CHIN C H, CHEN S H, WU H H, et al. cytoHubba: identifying hub objects and sub-networks from complex interactome[J]. BMC Syst Biol, 2014,8 Suppl 4: S11.

[20]SZKLARCZYK D, GABLE A L, LYON D, et al. STRING v11: protein-protein association networks with increased coverage, supporting functional discovery in genome-wide experimental datasets[J]. Nucleic Acids Res, 2019,47(D1): D607-D613.

[21]YU G, WANG L G, HAN Y, et al. clusterProfiler: an R Package for Comparing Biological Themes Among Gene Clusters[J]. Omics A Journal of Integrative Biology,16(5): 284-287.

[22]BINDEA G, MLECNIK B, HACKL H, et al. ClueGO: a Cytoscape plug-in to decipher functionally grouped gene ontology and pathway annotation networks[J]. Bioinformatics, 2009,25(8): 1091-1093.

[23]MAERE S, HEYMANS K, KUIPER M. BiNGO: a Cytoscape plugin to assess overrepresentation of gene ontology categories in biological networks[J]. Bioinformatics, 2005,21(16): 3448-3449.

[24]LONG Q X, LIU B Z, DENG H J, et al. Antibody responses to SARS-CoV-2 in patients with COVID$19[\mathrm{~J}]$. Nat Med, 2020.

[25]AZKUR A K, AKDIS M, AZKUR D, et al. Immune response to SARS-CoV-2 and mechanisms of immunopathological changes in COVID-19[J]. Allergy, 2020.

[26]WANG F, NIE J, WANG H, et al. Characteristics of Peripheral Lymphocyte Subset Alteration in COVID-19 Pneumonia[J]. J Infect Dis, 2020,221(11): 1762-1769.

[27]QIN C, ZHOU L, HU Z, et al. Dysregulation of immune response in patients with COVID-19 in Wuhan, China[J]. Clin Infect Dis, 2020.

[28]DEFTEREOS S G, SIASOS G, GIANNOPOULOS G, et al. The Greek study in the effects of colchicine in COvid-19 complications prevention (GRECCO-19 study): Rationale and study design[J]. Hellenic J Cardiol, 2020.

[29]CONTI P, RONCONI G, CARAFFA A, et al. Induction of pro-inflammatory cytokines (IL-1 and IL-6) and lung inflammation by Coronavirus-19 (COVI-19 or SARS-CoV-2): anti-inflammatory strategies[J]. J Biol Regul Homeost Agents, 2020,34(2).

[30]SIDDIQI H K, MEHRA M R. COVID-19 illness in native and immunosuppressed states: A clinicaltherapeutic staging proposal[J]. J Heart Lung Transplant, 2020,39(5): 405-407.

[31]WANG D, HU B, HU C, et al. Clinical Characteristics of 138 Hospitalized Patients With 2019 Novel Coronavirus-Infected Pneumonia in Wuhan, China[J]. JAMA, 2020.

[32]LI T, LU H, ZHANG W. Clinical observation and management of COVID-19 patients[J]. Emerg Microbes Infect, 2020,9(1): 687-690.

[33]BOCCIA M, ARONNE L, CELIA B, et al. COVID-19 and coagulative axis: review of emerging aspects in a novel disease[J]. Monaldi Arch Chest Dis, 2020,90(2).

[34]GALLELLI L, ZHANG L, WANG T, et al. Severe Acute Lung Injury Related to COVID-19 Infection: A Review and the Possible Role for Escin[J]. J Clin Pharmacol, 2020.

[35]XU Z, SHI L, WANG Y, et al. Pathological findings of COVID-19 associated with acute respiratory distress syndrome[J]. Lancet Respir Med, 2020,8(4): 420-422.

[36]GUO T, FAN Y, CHEN M, et al. Cardiovascular Implications of Fatal Outcomes of Patients With Coronavirus Disease 2019 (COVID-19)[J]. JAMA Cardiol, 2020. 
[37]SHI S, QIN M, SHEN B, et al. Association of Cardiac Injury With Mortality in Hospitalized Patients With COVID-19 in Wuhan, China [J]. JAMA Cardiol, 2020.

\section{Legend:}

Figure 1: A. The three prescriptions compounds were screened for active ingredients by OB [?] 30\% and DL [?] 0.18. The effective compounds in the three prescriptions were 116, 133, and 145, respectively. B. Venn diagram of intersected targets of effective compounds and disease. C. The number of connections between the intersecting genes and the randomly selected genes in protein interaction was significant different $(\mathrm{P}$ $<0.001$ ). D. Herb compounds and disease targets action network. (a: Mahuang Decoction; b: Yinqiao Powder; c: Dayuan Decoction) E. The five key compounds of the three prescriptions screened according to the disease target network diagram with each prescription compounds.

Figure 2: A-C. MH, YQ and DY PPI network respectively. D-F. MH, YQ and DY Hub gene network diagram respectively. G-I. Go rich clustering analysis of the hub genes of MH, YQ and DY respectively.

Figure 3: The top ten Hub genes of the three prescriptions by MCC value.

Figure 4: The Go biological process enrichment of all the intersection genes between herbs compounds and disease.

Figure 5: Directed Acyclic Graph of the three prescriptions.

Figure 6: The barplots of the top 20 entries according to the KEGG pathway enrichment analysis.

Figure 7: Network diagrams of the main mechanisms of five key compounds in the treatment of viral pneumonia.

Figure 8: Docking simulation of 5 key compounds with 3CL of COVID-19 (A: Kaempferol; B: Luteolin; C: Naringenin; D: Quercetin; E: Wogonin; F: Ligand).

Figure 9: The network analysis workflow for understanding how Chinese Medicine functions on COVID-19, based on Chinese Medicine theory and biological mechanisms.
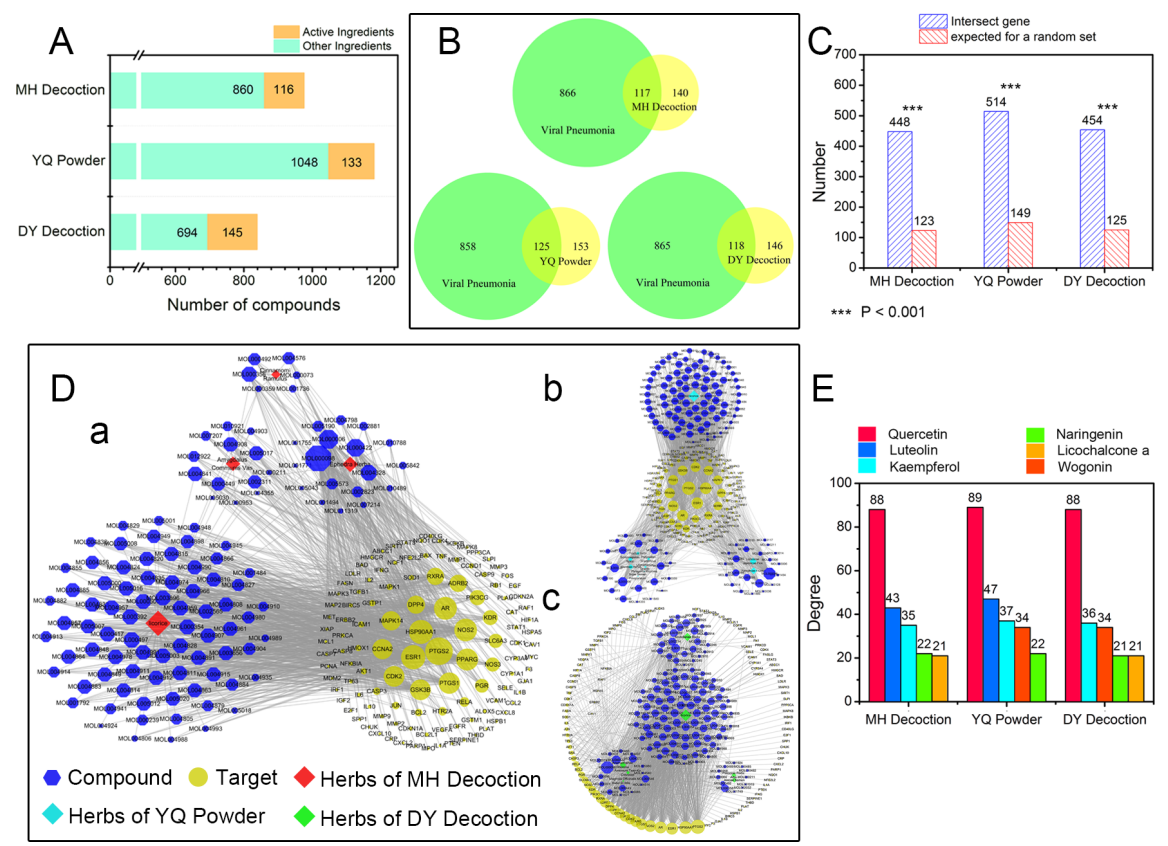

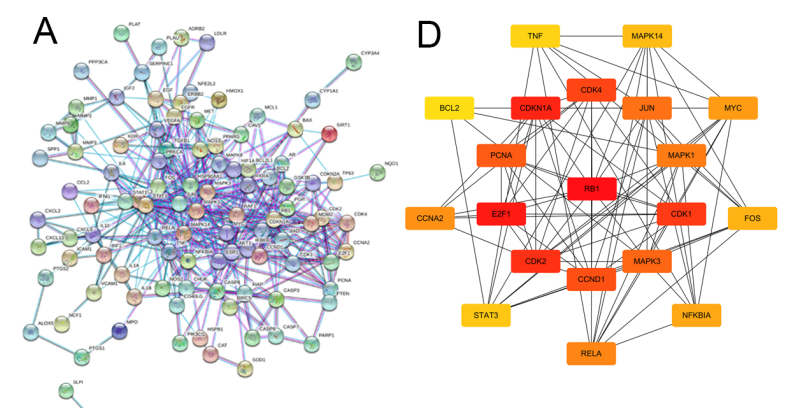

\section{G}
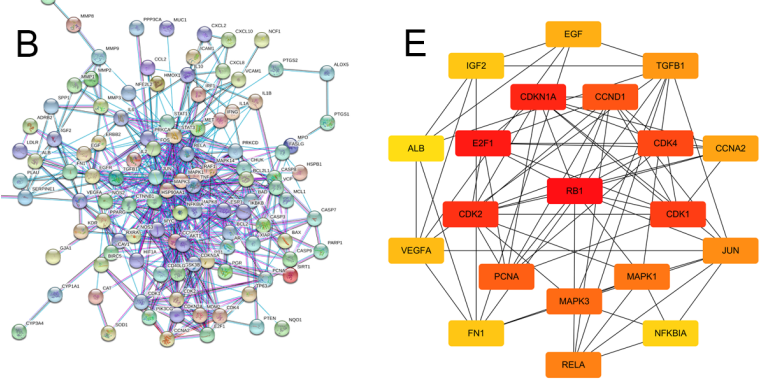

$\mathrm{H}$
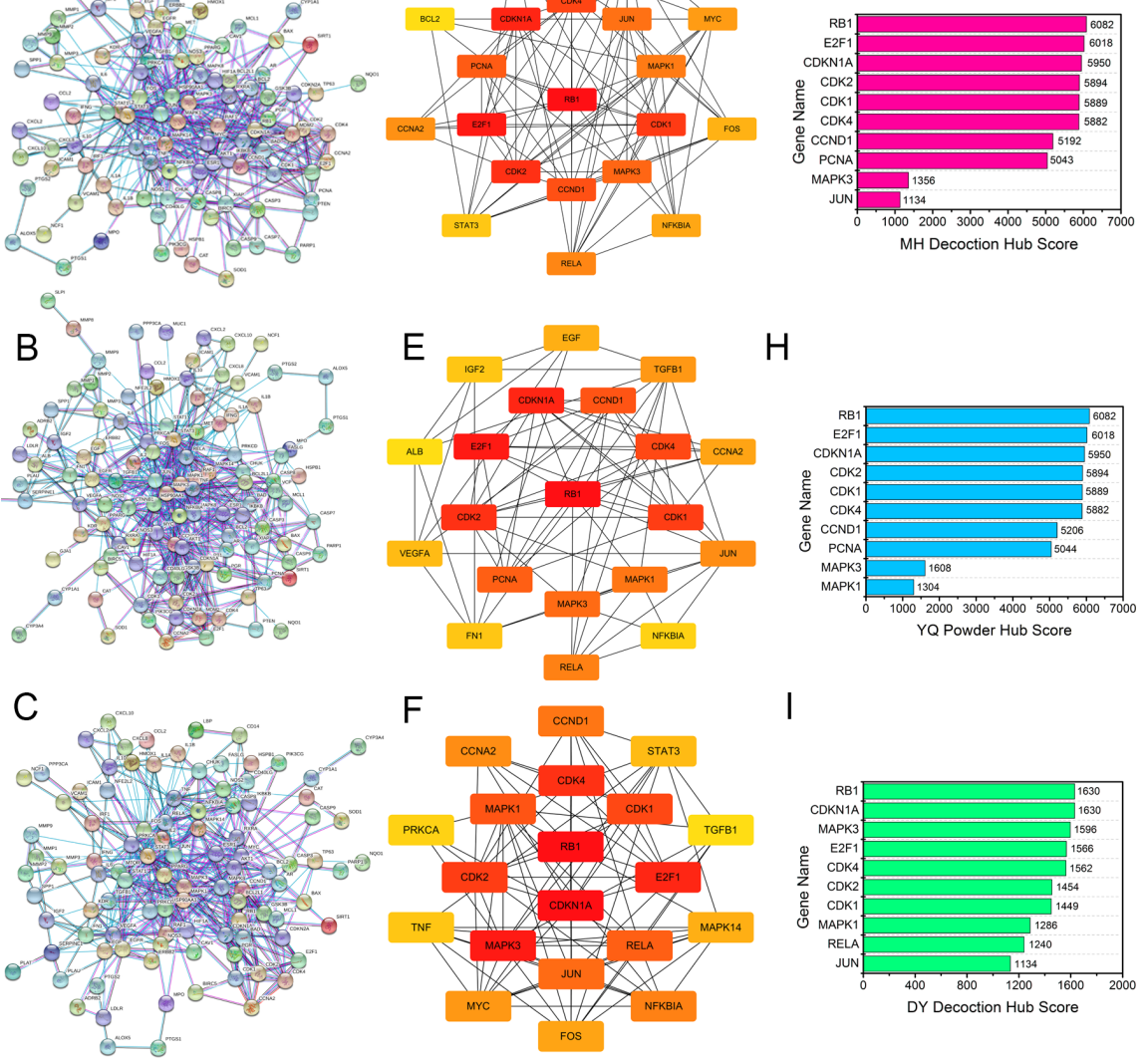

I
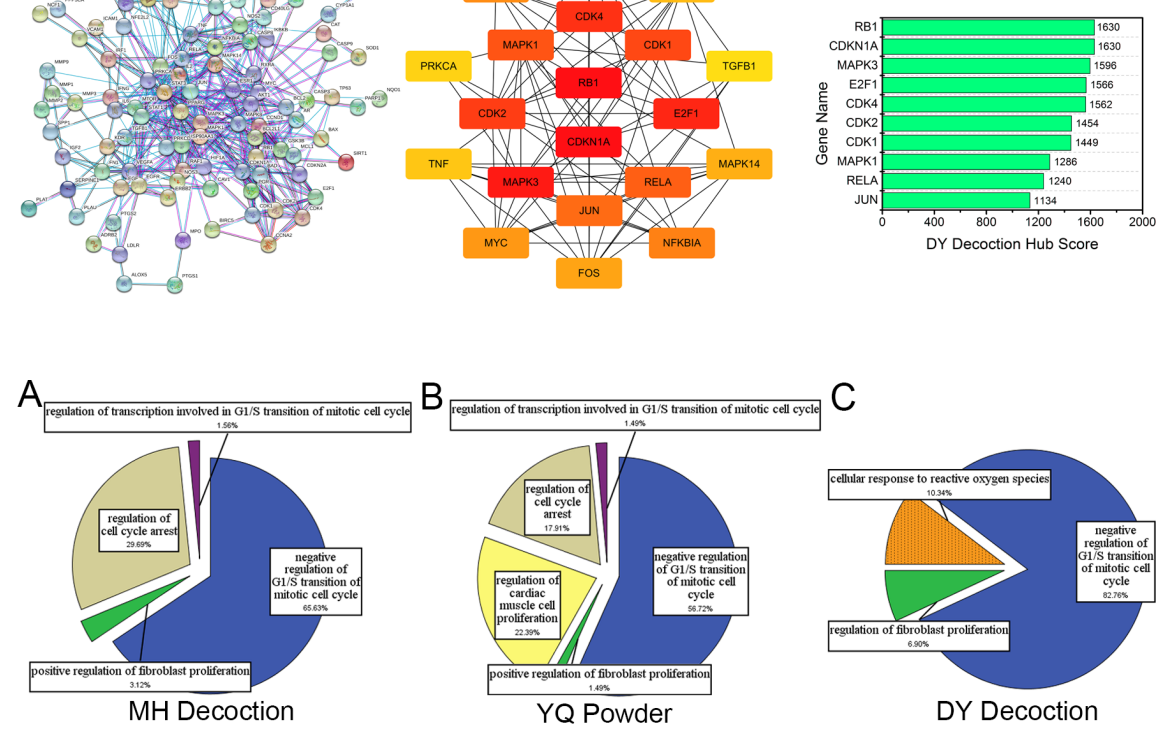
A

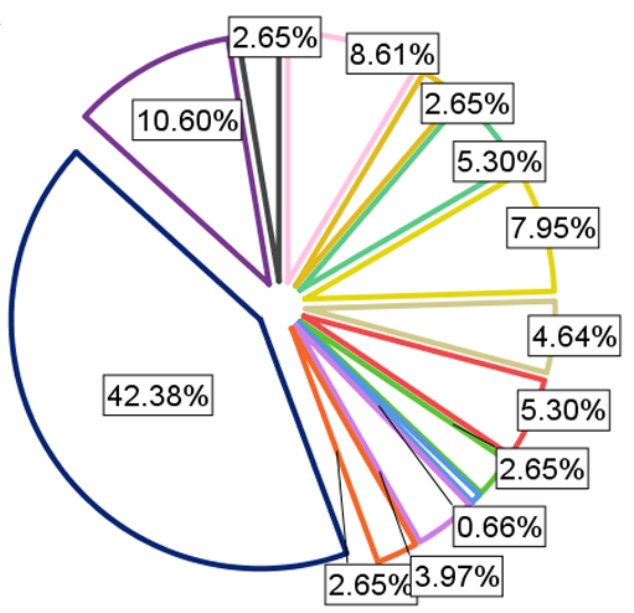

\section{B MH Decoction}
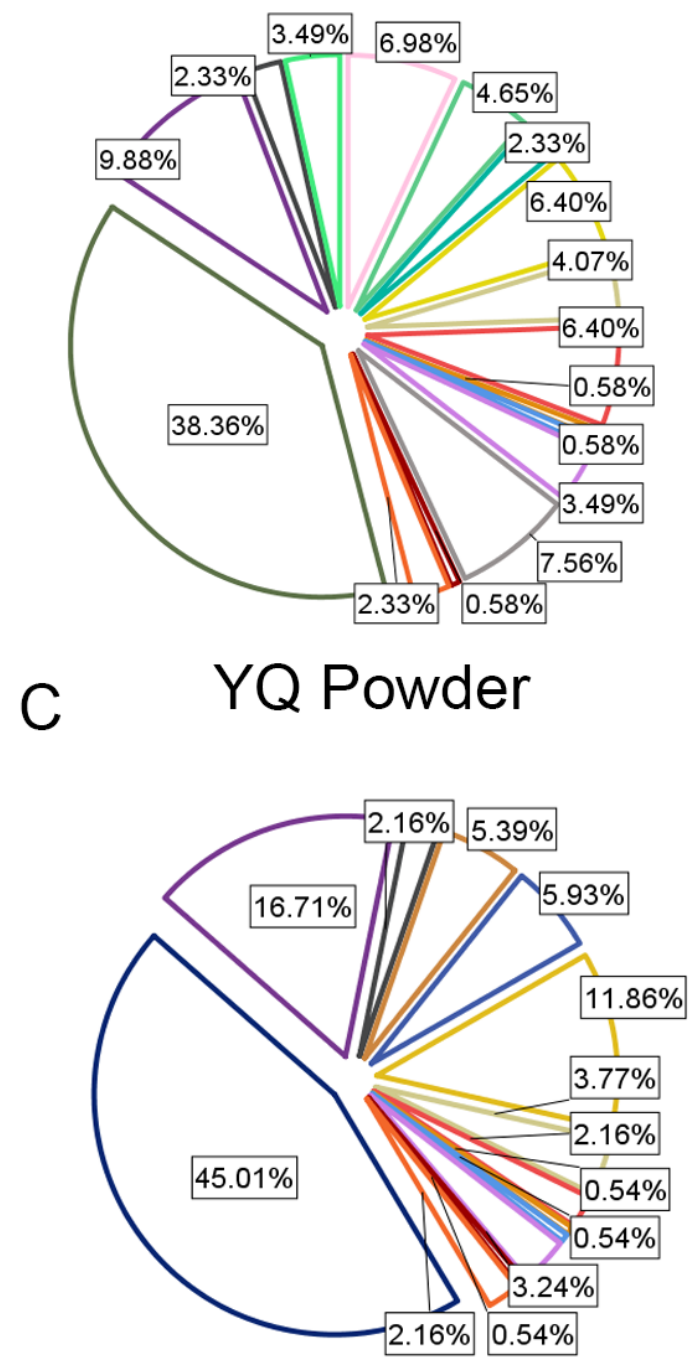

DY Decoction
Go type

cellular response to insulin-like

growth factor stimulus

$\square$ cellular response to nitric

oxide

connective tissue replacement

$\square$ involved in inflammatory

response wound healing

heat generation

mammary gland lobule

$\triangle$ development

$\square$ MAP kinase activity

$\square$ nitric-oxide synthase regulator

activity

$\square$ PERK-mediated unfolded

protein response

$\square$ positive regulation of cellular

respiration

positive regulation of

$\square$ transcription from RNA

polymerase 2 promoter in

response to hypox

$\square$ regulation of fever generation

regulation of immature $\mathrm{T}$ cell

proliferation

$\square$ removal of superoxide

radicals

GO term

cellular response to insulin-like factor stimulus

stimulus
connective tissue replacement involved in

inflammatory response wound healing

$\square$ epithelial cell differentiation involved in

prostate gland development

heat generation

mammary gland lobule development

$\square$ MAP kinase activity

negative regulation of extrinsic apoptotic

$\square$ signaling pathway via death domain

receptor

$\square$ PERK-mediated unfolded protein

response

$\square$ positive regulation of cellular respiration

$\square$ positive regulation of $\mathrm{MHC}$ class 2

biosynthetic process

$\square^{\text {positive regulation of superoxide anion }}$

generation

positive regulation of transcription from

$\square$ RNA polymerase 2 promoter in response

to hypoxia

$\square$ regulation of establishment of endothelia

$\square$ barrier

$\square$ regulation of immature $\mathrm{T}$ cell proliferation

$\square$ removal of superoxide radicals

$\square$ trachea formation

GO term

connective tissue replacement involved in inflammatory

response wound healing

mammary gland lobule

development

$\square$ MAP kinase activity

negative regulation of extrinsic

apoptotic signaling pathway via

death domain receptor

$\square$ PERK-mediated unfolded

protein response

positive regulation of cellular

respiration

$\square$ positive regulation of

superoxide anion generation

positive regulation of

transcription from RNA

polymerase 2 promoter in

response to hypoxia

$\square$ regulation of fever generation

$\square$ regulation of immature T cell

proliferation

removal of superoxide radicals

$\square^{T}$-helper cell lineage

commitment

$\square$ wound healing involved in

inflammatory response 

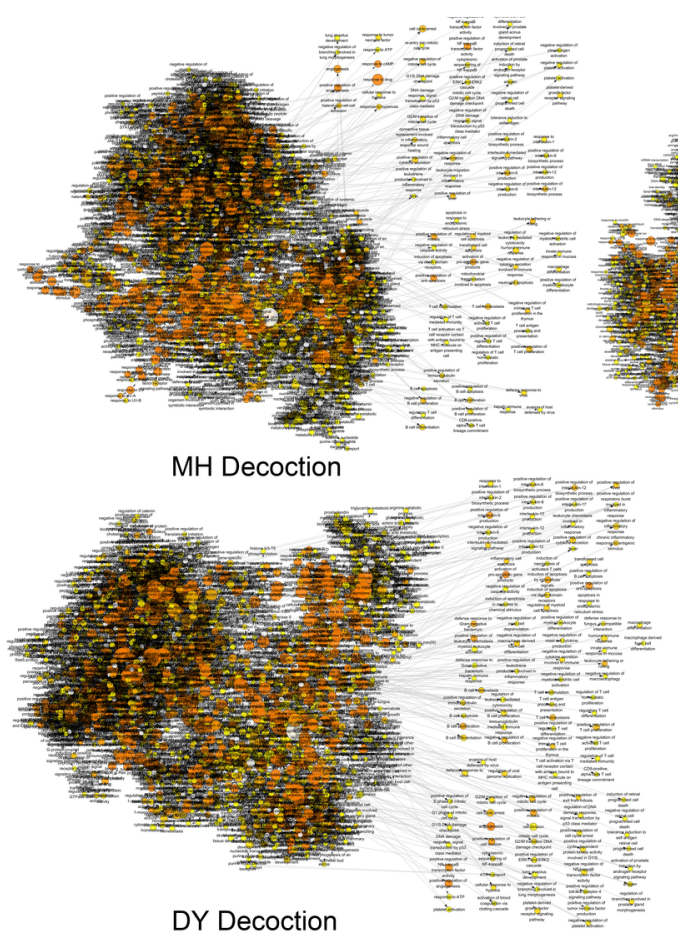
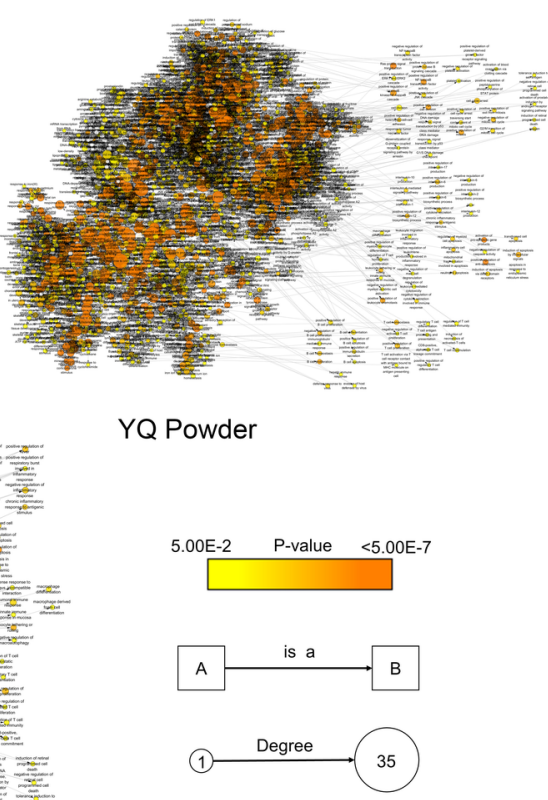
A

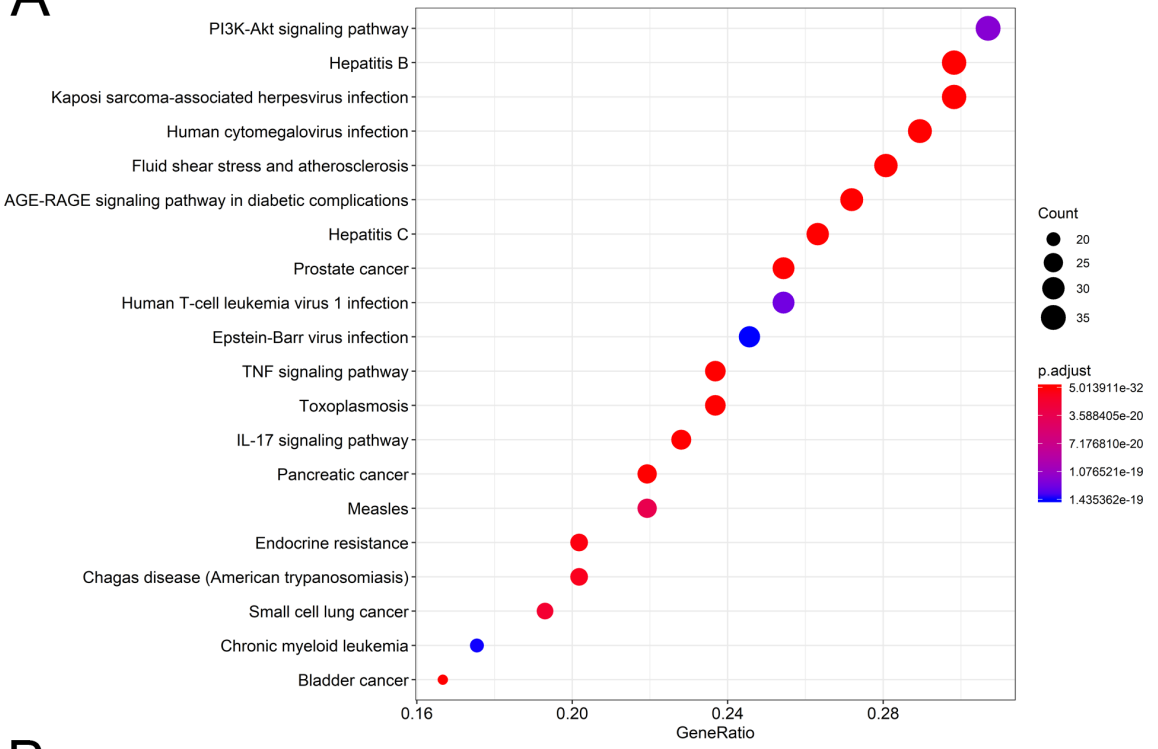

B

PI3K-Akt signaling pathway Hepatitis B-

Kaposi sarcoma-associated herpesvirus infection Human cytomegalovirus infection AGE-RAGE signaling pathway in diabetic complications Fluid shear stress and atherosclerosisHepatitis CProstate cancerProteoglycans in cancerTNF signaling pathwayToxoplasmosis Hepatocellular carcinomaIL-17 signaling pathway Measles Pancreatic cancer Chagas disease (American trypanosomiasis) Small cell lung cancerEndocrine resistanceC-type lectin receptor signaling pathwayBladder cancer -

C
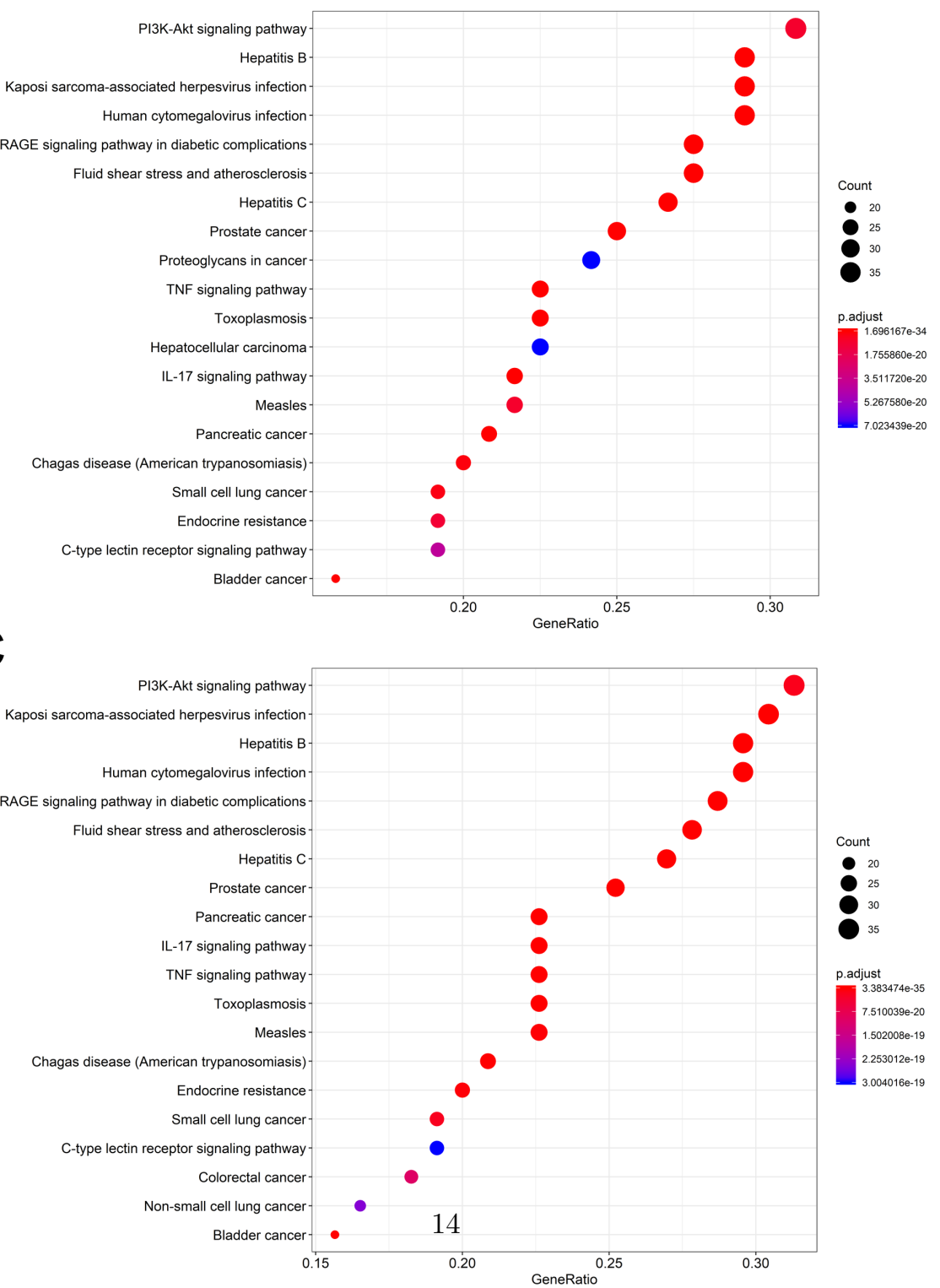
Five key compounds

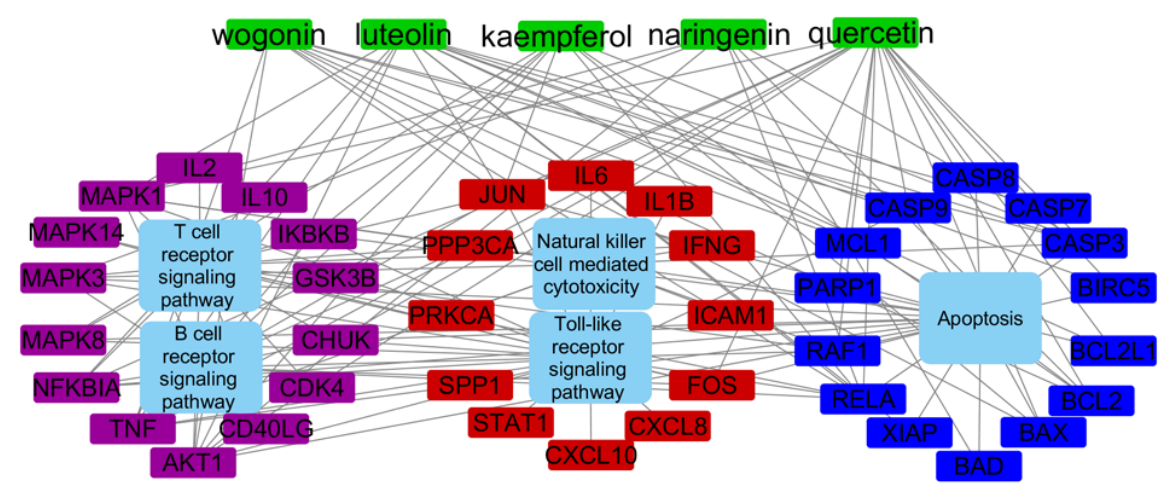

Adaptive Immunity Innate Immunity Apoptosis

\section{Viral Pneumonia}

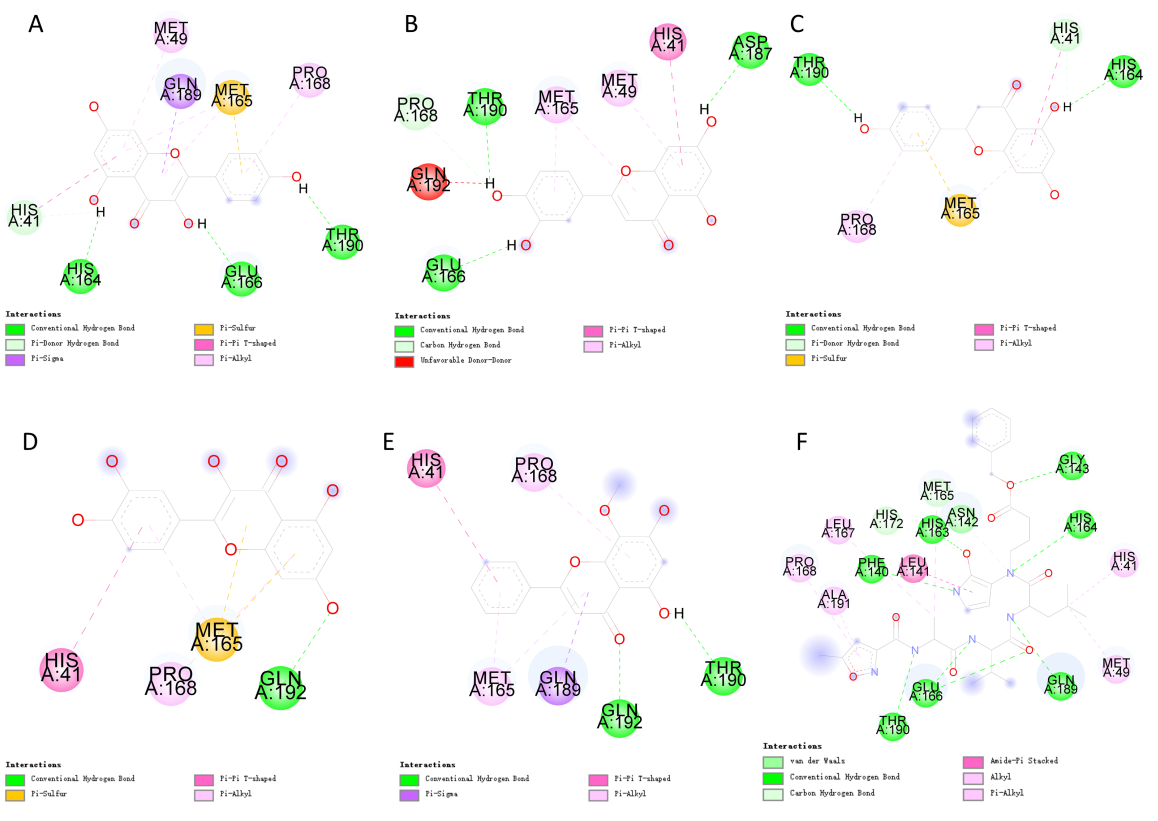




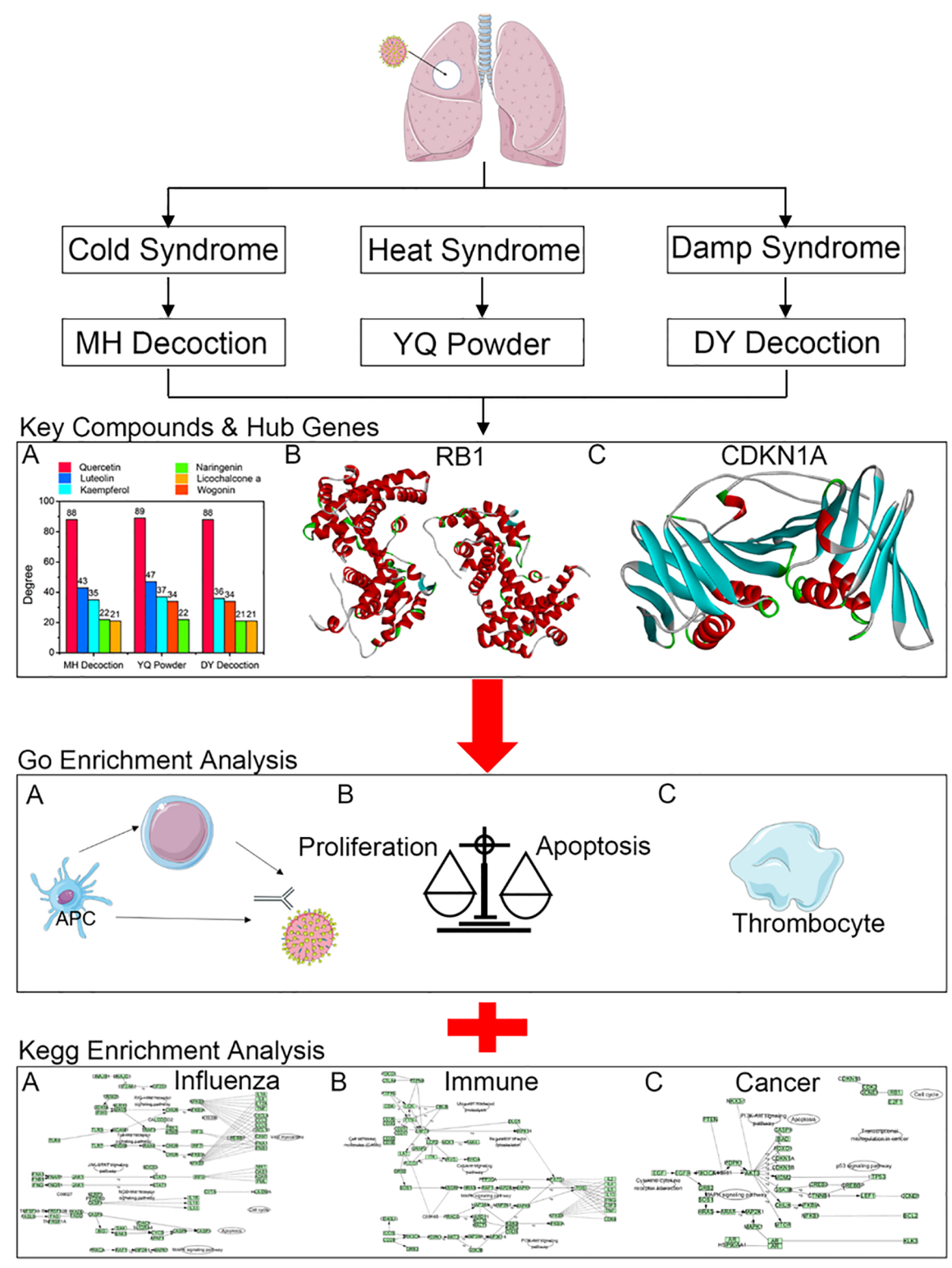

\section{Hosted file}

table1.docx available at https://authorea.com/users/333877/articles/459967-how-chinesemedicine-functions-on-covid-19-based-on-three-chinese-medicine-prescriptions-with-2201800-years-history

\section{Hosted file}

table2.docx available at https://authorea.com/users/333877/articles/459967-how-chinesemedicine-functions-on-covid-19-based-on-three-chinese-medicine-prescriptions-with-2201800-years-history

\section{Hosted file}

table3.docx available at https://authorea.com/users/333877/articles/459967-how-chinesemedicine-functions-on-covid-19-based-on-three-chinese-medicine-prescriptions-with-220- 
1800-years-history 